\title{
Comments on selected fundamental aspects of microarray analysis
}

\author{
Alessandra Riva ${ }^{\mathrm{a}, *}$, Anne-Sophie Carpentier ${ }^{\mathrm{a}}$, Bruno Torrésani ${ }^{\mathrm{b}}$, Alain Hénaut ${ }^{\mathrm{a}}$ \\ ${ }^{a}$ Laboratoire Génome et Informatique UMR 8116 Tour Evry2, 523 Place des Terrasses, 91034 Evry Cedex, France \\ ${ }^{\mathrm{b}}$ LATP, CMI, Université de Provence, 39 rue Joliot-Curie, 13453 Marseille Cedex 13, France
}

Received 26 May 2005; received in revised form 18 August 2005; accepted 18 August 2005

\begin{abstract}
Microarrays are becoming a ubiquitous tool of research in life sciences. However, the working principles of microarray-based methodologies are often misunderstood or apparently ignored by the researchers who actually perform and interpret experiments. This in turn seems to lead to a common over-expectation regarding the explanatory and/or knowledge-generating power of microarray analyses.

In this note we intend to explain basic principles of five (5) major groups of analytical techniques used in studies of microarray data and their interpretation: the principal component analysis (PCA), the independent component analysis (ICA), the $t$-test, the analysis of variance (ANOVA), and self organizing maps (SOM). We discuss answers to selected practical questions related to the analysis of microarray data. We also take a closer look at the experimental setup and the rules, which have to be observed in order to exploit microarrays efficiently. Finally, we discuss in detail the scope and limitations of microarray-based methods. We emphasize the fact that no amount of statistical analysis can compensate for (or replace) a well thought through experimental setup. We conclude that microarrays are indeed useful tools in life sciences but by no means should they be expected to generate complete answers to complex biological questions. We argue that even well posed questions, formulated within a microarray-specific terminology, cannot be completely answered with the use of microarray analyses alone.
\end{abstract}

(C) 2005 Elsevier Ltd. All rights reserved.

Keywords: Microarrays; Fundamental tools; FAQ section

\section{Introduction}

Microarrays have become one of the fundamental tools for biologists and great hopes are placed in their ability to answer all the questions asked by the researchers.

The amount of data created in an experiment is large and the nature of the data quantitative, two features a biologist is not necessarily used to or trained for. For the analysis of the data, the biologist has to choose from a rapidly increasing number of methods proposed in the literature, again, without necessarily having the knowledge and competence to do so. He therefore risks overestimating the power and capacity of the method (to provide him with the answers he is looking for).

\footnotetext{
* Corresponding author. Tel.: +331608738 63; fax: +33160873897

E-mail address: gucki@genopole.cnrs.fr (A. Riva).
}

This commentary wants to give the fundaments, which will allow the biologist to get out a maximum from microarrays, by understanding their nature and the principles of the statistical methods proposed to him.

For this we first give a brief introduction to the subject of microarrays, their origins, the different types and their application. We then examine the fundamental groups of methods used in the analysis of microarrays. Throughout we provide the reader with a list of papers allowing him to pursue the point further.

The FAQ section, which follows, contains the answers to questions related to the analysis of microarray data, often asked during the course taught by this laboratory (http://www.infobiogen.fr). This is another way to approach the subject and again, a list of publications for the interested reader is provided.

The last section leads us to consider which are the important aspects in the experimental setup, in function of the analysis methods discussed. 


\section{Fundamentals and basic terminology}

\subsection{General introduction to microarrays}

A microarray consist of a solid support on which a series of DNA segments is arranged and fixed in a regular pattern. These segments are incubated with a labelled nucleic acid sample. When a nucleic acid sequence in the sample is complementary to a DNA segment present on the support, it will bind and hybridize to this, specific segment. This hybridization is recorded and analyzed.

\subsubsection{The historical background}

As Jordan (2002) points out, DNA arrays were already being used in the seventies, in the form of dot blots and slot blots. Ekins and co-workers developed microspot fluorescent immunoassays in the late eighties and early nineties, proving that the sensitivity of these miniaturized assays was comparable to that of "macroscopic" ones and introducing the concept of microarray (Ekins, 1989; Ekins et al., 1990; Ekins and Chu, 1991). The concept of miniaturization was also applied to DNA arrays, using two different approaches. One was to deposit the DNA (or complementary DNA) on glass plates, leading to the first publication of a gene expression microarray article in 1995 (Schena et al., 1995). The second approach was that of the oligonucleotide array, where the DNA is directly synthesized onto the support (Fodor et al., 1991; Southern et al., 1992).

\subsubsection{Today's microarrays}

In the following, "probe" denotes the immobilized DNA on the support and "target" the mobile DNA, cDNA or mRNA. Some authors, however, use the terms the other way round. The supports used for microarrays today are glass (microscope) slides (nylon) membranes or silicon chips.

The material fixed on the support ("probe") can be:

a. DNA, representing coding sequences or, more generally, pieces of genomic DNA.

b. Complementary DNA, obtained from the mRNA of specific genes or expressed sequence tags (ESTs); the latter is usually used for organisms not yet completely sequenced.

c. Oligonucleotides; in the case of oligonucleotide arrays the oligos are synthesized directly onto a silicon chip; this process has been pioneered by Affymetrix (see Lipshutz et al. (1999) for a comprehensive review on oligonucleotide arrays).

The mobile "target" can be:

a. DNA.

b. Complementary DNA (cDNA), obtained from mRNA by reverse transcriptase-PCR (RT-PCR).

c. mRNA; this can be used although cDNA is generally preferred.

A hybridization mixture is obtained by labelling the target fluorescently or radioactively. This mixture is then incubated with the prepared microarray and allowed to hybridize with the probe. Finally, the resulting signal intensity, which correlates with the amount of captured probe, is measured, stored in a computer and then analyzed.

Recently, efforts have been made to extend the microarray technology to the field of proteins. The interested reader may refer to the review written by Templin et al. (2002) for a comprehensive introduction to this field. For further information on microarray technology, the reader may refer to recent review articles (Barrett and Kawasaki, 2003; Vrana et al., 2003); he may also refer to a related NCBI web page (http://www.ncbi.nlm.nih.gov/About/ primer/microarrays.html).

\subsection{Applications}

Microarrays can be used for a variety of purposes, including the detection of mutations, DNA sequencing and the analysis of gene expression. Microarrays allow measuring the expression levels of thousands of genes at the same time and this opens the possibility to identify differentially expressed genes (Callow et al., 2000) and to cluster those genes sharing similar expression patterns (Heyer et al., 1999). They have become a widespread tool for analyzing the relative transcription levels of genes.

The fields microarrays are being used in are numerous and constantly growing, some examples being:

a. clinical medicine (see Joos et al. (2003) for a review on this subject);

b. the study of the cell-cycle (see for example McCune and Donaldson (2003));

c. the study of the circadian rhythm in animals (see for example Stanewsky (2003)) and plants (see for example Davis and Millar (2001));

d. the study of plant metabolism (see for example Hirai et al. (2004)).

They are also being used to elucidate the role of noncoding sequences, for example, the role of some promoter regions, by integrating expression profiles with the information on promoter sequence similarity (Bussemaker et al., 2000; Park et al., 2002). Heterologous hybridization to cDNA microarrays is gaining in popularity and is, for example, used in order to elucidate the molecular basis of complex traits in "non-traditional model systems" (Renn et al., 2004).

As different as these applications may seem, the aim of the experiments is one of the following:

a. To find the genes which indicate a phenomenon (not necessarily at the origin of the phenomenon, but an indicator of it: expression change correlated with the phenomenon).

b. To find the genes which are at the origin of the phenomenon under investigation.

In the first case, the researcher will need to find genes whose expression levels change considerably, few in numbers and that can be preferably used in antibody assays (still 
cheaper and faster to set up than microarrays) (Deutsch, 2003); an analysis of the microarray data will generally be sufficient in order to identify the genes.

The work done by Sekowska et al. (2001) and Oshima et al. (2002) are examples for the second case and we will come back to them in the course of the commentary; here, an analysis of the microarray data is not enough to find the genes at the origin of a phenomenon (and only these): it is necessary to combine the results of the microarray analysis with information from other sources, such as the genomic and the purely biological fields (Jarvis et al., 2004; Hirai et al., 2004; Riva et al., 2004). This is something important to bear in mind and will be discussed at various points of the commentary.

\section{Data representation and analysis}

\subsection{The raw data}

The microarray data used in the following stem from experiments on the sulphur metabolism of $B$. subtilis (Sekowska et al., 2001) and are freely available at http://195.221.65.10:1234/ carpenti/. The experiments were carried out using Panorama nylon filters $B$. subtilis gene arrays (Sigma-GenoSys Biotechnologies); each array contains all of $B$. subtilis' genes and one gene is represented by one spot. Each gene spot is represented twice on the array.

The aim of these experiments was to identify the genes differentially expressed when the bacteria are grown with methionine ("met") or methyl-thioribose ("mtr") as sulphur source. The experiments followed a fully crossed factorial design with four factors (sulphur source, day of experiment, amount of RNA used and duplicate of each spot). The data (raw levels of expression) were gathered in an array of 4107 rows (all B. subtilis genes) and 16 columns (experimental conditions). The minimum value was 213 , the maximum value 13,455 , with two thirds of the data having a value below 800 . Note that each factor has only two states: all factors are binary (see Fig. 1).

\subsection{The data table and some preliminary considerations and manipulations}

It is natural to want to represent the data in a graph. We obtain one (and only one!) graph, with $N$ dimensions (corresponding to the $N$ experimental conditions), a cloud in an $\mathrm{N}$-dimensional space. As we are not good at coping with drawings having more than two dimensions (three still works well on a computer screen), we are obliged to take the columns 2 by 2 (i.e. one experimental condition versus another).

Note that when you draw a graph by hand, you will automatically try to maximize the use of the paper: you look at the minimum and maximum values for both variables, and define the scale accordingly. The machine will do the same. In both cases, the data are transformed through a change of variable: $1 \mathrm{~cm}$ on the graph corresponds to $X$ units of the original variable (a linear transformation).

\subsubsection{Translation}

This is an operation which in itself does not pose a problem, as one is interested in the relative position of the points to each other: the aim is to find the points that are far away relative to the main body of the cloud, which means that the reference frame used to look at the cloud does not really have much importance. However, the translation may create complications when it consists in bringing a lot of the values close to zero followed by taking the log of the data, something discussed in the next section.

\subsubsection{Normalization}

Note that drawing a graph or letting a spreadsheet (like MS-Excel) draw the graph, implicitly presumes that the sum of the signal does not change in function of the experimental conditions; one allows the data to be normalized. By doing this, one has presumed that the total of the signal in each column is the same: total signal of column $1=$ total signal of column 2. This is justified when three conditions are fulfilled: firstly, more than $90 \%$ of the genes do not care about the experiment, i.e. do not change expression in function of the different experimental conditions; in that case one can indeed presume that the total quantity of cDNA (and therefore of the mRNA) is the same. Secondly, the number of genes analyzed has to be large: this is a way to make sure that the majority of the genes do not change expression in function of the different experimental conditions. Thirdly, the overall intensity change of up- and down-regulated genes is similar. The three conditions are fulfilled in our example, but they would not be in, say, the temporal analysis of mRNA decay. The reader is referred to the work of Stoyanova et al. (2004) for some interesting considerations on this subject, as well as to the work

\author{
Sulphur Source \\ Day of experiment \\ Protocol \\ Duplicate
}

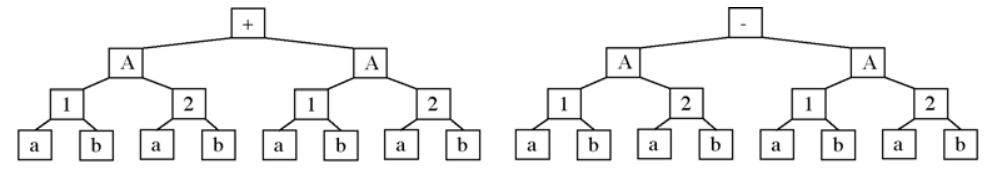

Fig. 1. Experimental design of the transcriptome analysis on Bacillus subtilis (Sekowska et al., 2001). The experimental setup follows a fully crossed factorial design. In the case of Sekowska et al. (2001) the quantity of RNA used for the RT-PCR differed between the two protocols. Note that changing the protocol (a different quantity of RNA or labelling with Cy3 rather than Cy5) or having duplicats for each gene on the array are all technical factors which increase the workload without adding any biologically pertinent information. It is preferable to increase the number of states for the biological factors, in the above case an additional sulphur source or an additional experimental day. 
of Zhao et al. (2005) who propose a normalization procedure for data not fulfilling the above conditions.

Instead of just looking at the minimal and maximal values in order to best represent the graph, it is advisable to calculate the means and variance for each experimental condition: in the first case the estimates are based on two points only ( $\mathrm{min}$ and max) per experimental condition, in the second case the estimate is made using all points. If these are numerous, the result is more stable.

\subsection{Graphic exploration}

\subsubsection{Preliminary considerations}

As we said, we are forced to take the columns 2 by 2 , which means that we will look at projections of our single cloud on the different planes.

What are we looking for? Presuming that the three abovementioned conditions are fulfilled, at least $90 \%$ of the genes analyzed will not change expression under the different experimental conditions. This means that on the graph one would see them all lying on one line, if it was not for the noise: the noise is responsible for making those points look more like a cigar which is the wider the more noise there is. The remaining $10 \%$ of the genes will change expression; they have an atypical behaviour and will not lie on the line (the cigar) but be apart. These genes that are apart from the main body of the cloud are the ones the biologist is interested in. Note that having the $90 \%$ of the point lying on a line is an ideal case, the "cigar" being the reality; so one tries to find that line (which describes $90 \%$ of the genes) somehow.

How do we describe those $90 \%$ of the data? How do we determine the line? Various options are available:

(a) One can try to draw it by hand.

(b) Calculate the linear regression. This is not such a good idea as there are two lines of regression ( $x$-axis versus $y$-axis and vice versa) and they are not identical except when all the points lie on the same line.

(c) Use methods that are more sophisticated.

The methods all presume that the cloud follows a Gaussian distribution, or at least a unimodal and symmetrical one. They also need some pre-processing of the data, for two reasons:

(a) The fact that the data often consist of a very large amount of small values and a few, extreme points, something which affects most data analysis techniques strongly (Chiappetta et al., 2004).

(b) Some effects being studied may have a multiplicative behaviour.

To solve the first of these problems, taking the log, the square (or cubic or fifth etc) root or the hyperbolic tangent are all possible and generally accepted methods (see Fig. 2), whilst for the second problem taking the log is preferable (Chiappetta et al., 2004; Hoyle et al., 2002; Thygesen and Zwinderman, 2004; Tusher et al., 2001).

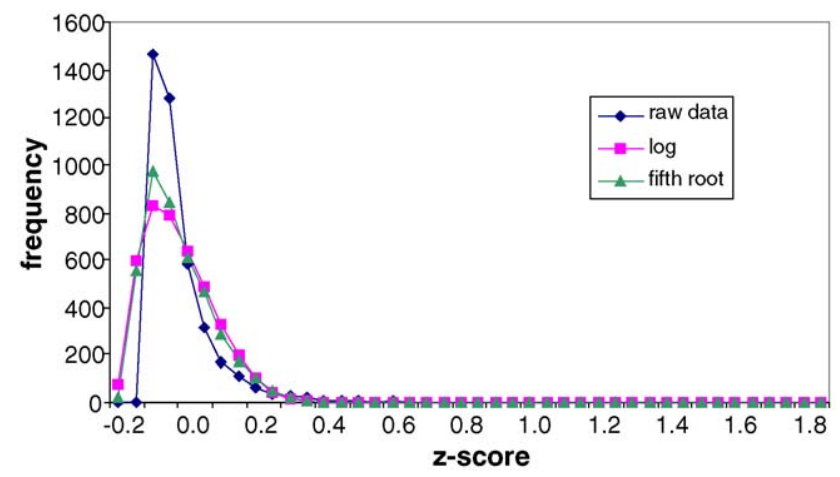

Fig. 2. Effect of different pre-processing methods on the data distribution. The figure shows the effect different pre-processing methods have on the data distribution. Shown are the distributions $(\bullet)$ of the raw data, ( $\square$ ) after having taken the $\log$ and $(\boldsymbol{\Lambda})$ after having taken the fifth root. As can be seen, either operation brings the distribution closer to a Gaussian one.

As we mentioned in the section above, the reference frame used to look at the cloud does not really matter and making a simple translation does not in itself pose a problem. One does need to be careful, though: making a simple translation is indeed no problem, nor is taking the log. However, when executing both operations, one needs to be cautious: if the translation consists in bringing a lot of the values close to zero, taking the log afterwards will create a distortion in the cloud of points: one has just created a whole package of data with values going towards minus infinity. This means that in trying to take care of the problem of the points at the far right (few points with very large values) by taking the log, the result is worse than the starting point. Note that when executing the two operations in the inverse order (first log, then translation) the problem is not created.

We come back to the graphs, which are just many projections on different planes of ONE cloud. A brief look at the general shape of each cloud projection is worthwhile. If a cloud resembles a fat cigar, a lot of genes have considerably changed expression. If, on the other hand, the cloud resembles a line, the great majority has not changed expression (see Fig. 3 for two examples). The "cigar" may also be bent or twisted. In this case the readings were taken outside the linear range of the machine, an issue discussed in Section 4.1.7. We can be faced with a problem: taking the columns 2 by 2 , the number or graphs increases very rapidly when increasing the number of experimental conditions: in our example we have 16 columns which means we need to look at $16 \times 15 / 2$ i.e. 120 graphs. Evaluating them all in detail becomes a bit tedious.

Thus, we need to find ways to reduce the number of graphs we have to examine. To do this, we need to decide, from which point of view we want to look at the cloud, which has to be translated into a mathematical criterion. This implies that there will be a change (rotation) of the reference frame.

\subsubsection{By hand (with a spreadsheet)}

With "by hand", we refer to the fact that the calculations are extremely simple. As the calculations have to be repeated 

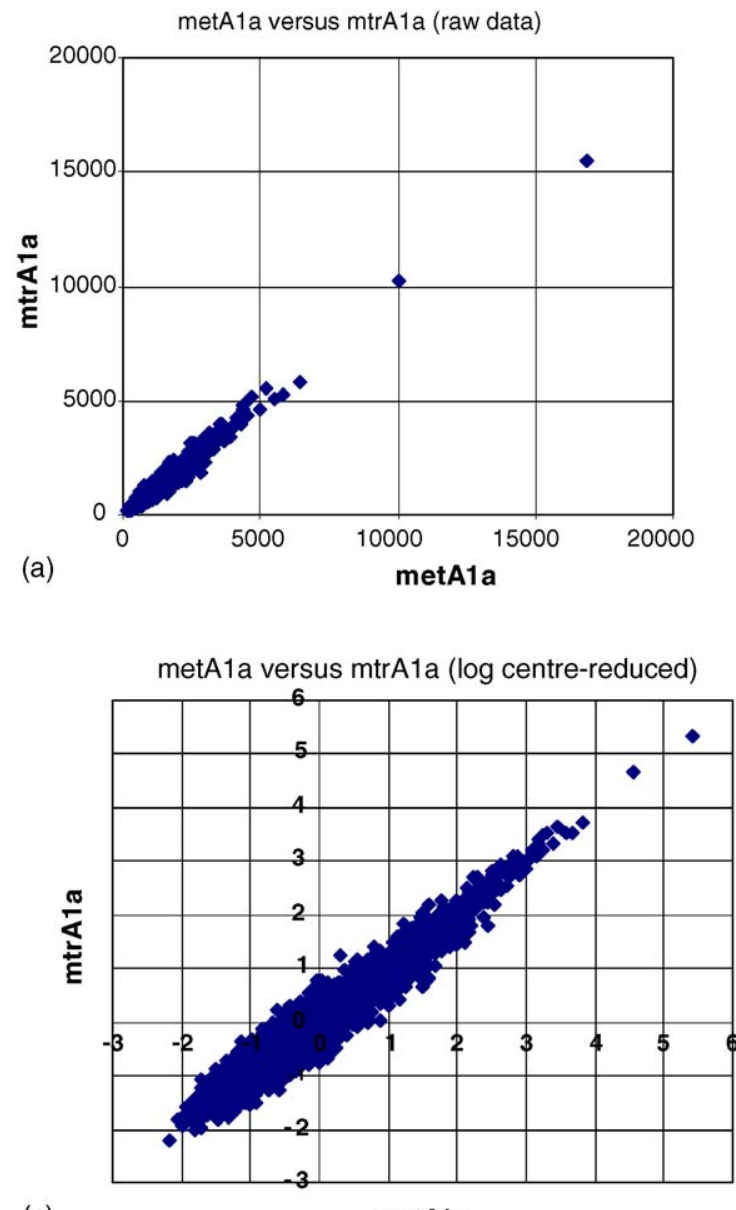

(c)

metA1a
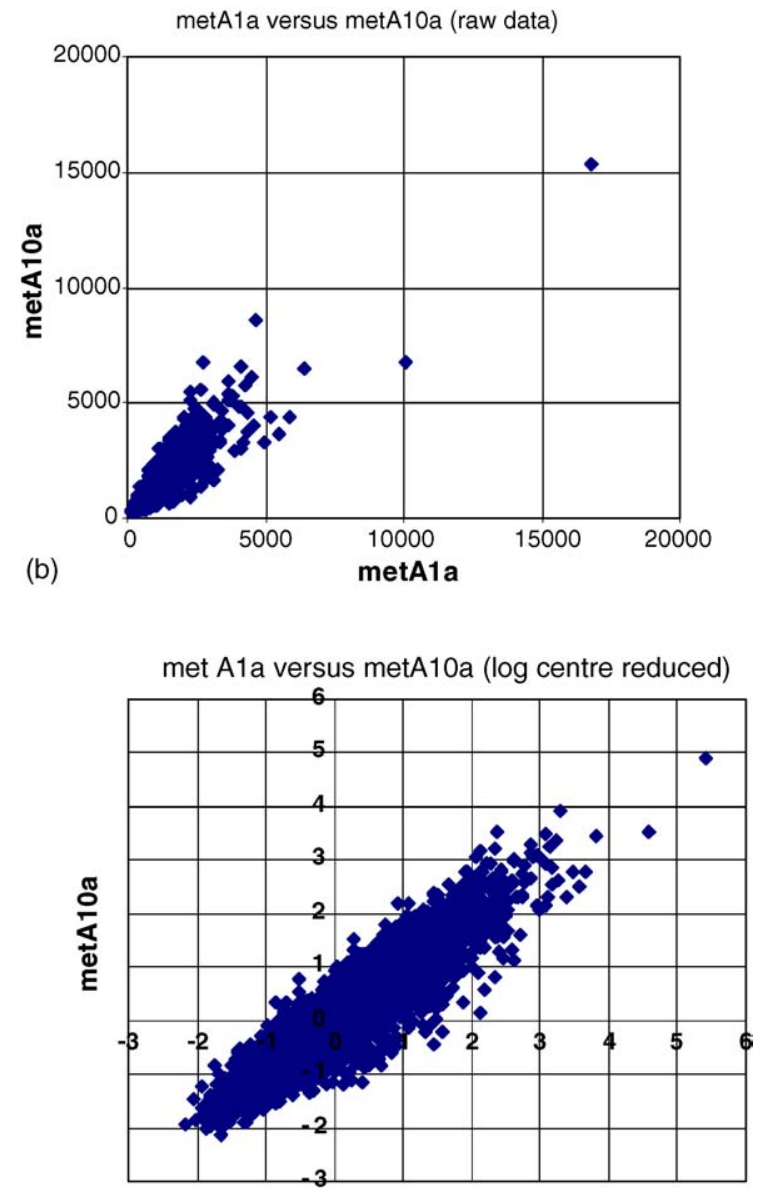

(d)

metA1a

Fig. 3. Projections of the data on different planes. In all four figures, each axis corresponds to an experimental condition: (a and c) metA1a vs. mtrA1a; (b and d) metAla vs. metA10a (see Fig. 1 for the nomenclature). (a) and (b) show projections of the raw data, in (c) and (d) the data are log centre-reduced. Log centre-reducing the data has brought the few points which are far away from the main body in (a) and (b) closer in (c) and (d). Note how the space is more efficiently used in (c) and (d). The points in the two left-hand pictures form a narrower "cigar", indicating that fewer genes have changed expression than on the right-hand side.

for each gene, though, the number of calculations is such as to make handing the job over to a spreadsheet a practical alternative.

The only reasonable option to reduce the 120 little pictures means concentrating on the expression changes caused by each single factor being studied, in our case four. For this we calculate the mean expression for each gene; this will be the $x$-value. Then, for a given factor like sulphur, we calculate the sum of all met values and subtract from it the sum of all mtr values, which gives us the $y$-value.

This is done for all four factors. Note that we have changed the reference frame; this calculation, which is done instinctively by hand, can be formalized and done via a matrix, called "mixing matrix": it allows to change from the old reference frame to the new one and is shown in Table 1.

We obtain four graphs, one for each factor; we then look for genes that are far away from the main body of the cloud. Fig. 4 shows the graph obtained for the factor sulphur. Executing this operation, each experimental condition is given the same weight and the criterion chosen to look at the cloud is "one factor per graph".

\subsection{3. $P C A$}

A more sophisticated approach is the principal component analysis. Pearson first introduced it in 1901. The reader may refer to the work by Stoyanova et al. (2004) for a comprehensive introduction to the subject and to Kendall et al. (1983) for a technical presentation.

Here, the criterion chosen to look at the cloud is to maximize the variances along the axes of the reference frame. There are numerous softwares that do this job and which supply us with the mixing matrix, which in PCA's case is called eigenvector matrix, shown in Table 1. This matrix allows us to change from the old reference frame to the new one; it gives us for each of the new axes (in the table: the columns) the coefficient with which we have to multiply each gene's value in a given experimental condition (in the table: the lines) in order to obtain its new coordinates (see legend of Table 1). 


\section{Axis}

\begin{tabular}{|c|c|c|c|c|c|c|c|c|c|c|c|c|c|c|c|c|c|}
\hline & & & & & & & & & & & & \\
\hline & & $\begin{array}{r}\text { Mean } \\
\text { expression }\end{array}$ & $\begin{array}{l}\text { Effect of } \\
\text { protocol }\end{array}$ & $\begin{array}{r}\text { Effect of } \\
\text { day }\end{array}$ & $\begin{array}{r}\text { Effect of } \\
\text { sulphur } \\
\text { source }\end{array}$ & $\begin{array}{r}\text { Effect of } \\
\text { duplicate }\end{array}$ & & & & & & & & & & & \\
\hline \multirow{14}{*}{ 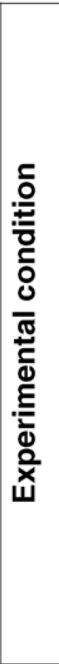 } & metA1a & 0.250 & 0.250 & -0.250 & 0.250 & -0.250 & & & & & & & & & & & \\
\hline & metA1b & 0.250 & 0.250 & -0.250 & 0.250 & 0.250 & & & & & & & & & & & \\
\hline & metB1a & 0.250 & 0.250 & 0.250 & 0.250 & -0.250 & & & & & & & & & & & \\
\hline & metB1b & 0.250 & 0.250 & 0.250 & 0.250 & 0.250 & & & & & & & & & & & \\
\hline & nætA10a & 0.250 & 0.250 & -0.250 & 0.250 & -0.250 & & & & & & & & & & & \\
\hline & metA10b & 0.250 & -0.250 & -0.250 & 0.250 & 0.250 & & & & & & & & & & & \\
\hline & metB10b & 0.250 & -0.250 & 0.250 & 0.250 & 0.250 & & & & & & & & & & & \\
\hline & mtrA1a & 0.250 & 0.250 & -0.250 & -0.250 & -0.250 & & & & & & & & & & & \\
\hline & mtrA1b & 0.250 & 0.250 & -0.250 & -0.250 & 0.250 & & & & & & & & & & & \\
\hline & mtrB1a & 0.250 & 0.250 & 0.250 & -0.250 & -0.250 & & & & & & & & & & & \\
\hline & mtrB1b & 0.250 & 0.250 & 0.250 & 0.250 & 0.250 & & & & & & & & & & & \\
\hline & mtrA10a & 0.250 & -0.250 & -0.250 & -0.250 & -0.250 & & & & & & & & & & & \\
\hline & mtrA10b & 0.250 & -0.250 & -0.250 & -0.250 & 0.250 & & & & & & & & & & & \\
\hline & mtrB10a & 0.250 & -0.250 & 0.250 & -0.250 & -0.250 & & & & & & & & & & & \\
\hline & & \multicolumn{16}{|c|}{ Axis } \\
\hline & & 1 & 2 & 3 & 4 & 5 & 6 & 7 & 8 & 9 & 10 & 11 & 12 & 13 & 14 & 15 & 16 \\
\hline & eigenvalue & 94.64 & 2.26 & 1.01 & 0.56 & 0.36 & 0.29 & 0.26 & 0.18 & 0.16 & 0.05 & 0.05 & 0.05 & 0.04 & 0.04 & 0.03 & 0.02 \\
\hline \multirow{14}{*}{ 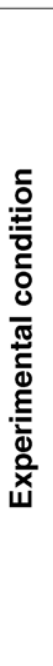 } & metA1a & 0.250 & 0.307 & -0.164 & -0.114 & 0.336 & -0.004 & -0.310 & 0.179 & 0.349 & -0.146 & -0.130 & 0.406 & -0.228 & 0.429 & -0.020 & -0.017 \\
\hline & metA1b & 0.249 & 0.312 & -0.166 & -0.131 & 0.366 & 0.075 & 0.157 & 0.252 & 0.347 & 0.131 & 0.078 & -0.429 & 0.262 & -0.413 & 0.001 & 0.026 \\
\hline & metB1a & 0.252 & 0.179 & 0.115 & 0.365 & 0.124 & 0.306 & -0.301 & -0.280 & -0.158 & 0.172 & 0.629 & -0.094 & -0.019 & 0.132 & 0.052 & 0.028 \\
\hline & metB1b & 0.251 & 0.181 & 0.107 & 0.369 & 0.153 & 0.425 & 0.211 & -0.246 & -0.203 & -0.170 & -0.592 & 0.072 & 0.003 & -0.140 & -0.036 & -0.027 \\
\hline & metA10a & 0.249 & -0.256 & -0.326 & 0.211 & 0.200 & -0.429 & -0.183 & -0.129 & -0.186 & 0.372 & -0.309 & -0.254 & 0.193 & 0.264 & 0.082 & 0.028 \\
\hline & metA10b & 0.249 & -0.255 & -0.328 & 0.192 & 0.260 & -0.303 & 0.315 & -0.027 & -0.146 & -0.388 & 0.325 & 0.261 & -0.198 & -0.276 & -0.081 & -0.031 \\
\hline & metB10a & 0.250 & -0.254 & 0.263 & -0.232 & 0.126 & 0.086 & -0.322 & 0.311 & -0.322 & -0.041 & -0.070 & -0.072 & -0.146 & -0.163 & -0.330 & 0.505 \\
\hline & metB10b & 0.250 & -0.253 & 0.259 & -0.236 & 0.178 & 0.180 & 0.170 & 0.358 & -0.283 & 0.064 & 0.067 & 0.092 & 0.171 & 0.180 & 0.335 & -0.508 \\
\hline & mtrA1a & 0.248 & 0.334 & -0.083 & -0.365 & -0.224 & -0.182 & -0.173 & -0.256 & -0.270 & 0.108 & 0.003 & 0.432 & 0.363 & -0.310 & 0.093 & 0.018 \\
\hline & mtrA1b & 0.248 & 0.331 & -0.087 & -0.370 & -0.189 & -0.093 & 0.321 & -0.159 & -0.277 & -0.089 & 0.029 & -0.407 & -0.399 & 0.305 & -0.086 & -0.028 \\
\hline & mtrB1a & 0.251 & 0.156 & 0.220 & 0.311 & -0.360 & -0.291 & -0.164 & 0.272 & 0.049 & -0.540 & 0.002 & -0.211 & 0.315 & 0.102 & 0.061 & -0.029 \\
\hline & mtrA10b & 0.249 & -0.240 & -0.352 & -0.030 & -0.342 & 0.369 & 0.195 & 0.052 & 0.150 & 0.053 & 0.087 & 0.108 & 0.352 & 0.262 & -0.471 & 0.045 \\
\hline & mtrB10a & 0.251 & -0.226 & 0.335 & -0.131 & -0.003 & -0.151 & -0.196 & -0.375 & 0.341 & 0.014 & -0.054 & -0.094 & -0.118 & -0.174 & -0.385 & -0.484 \\
\hline & mtrB10b & 0.251 & -0.225 & 0.327 & -0.140 & 0.041 & -0.057 & 0.310 & -0.322 & 0.358 & -0.037 & 0.036 & 0.067 & 0.101 & 0.164 & 0.376 & 0.491 \\
\hline
\end{tabular}




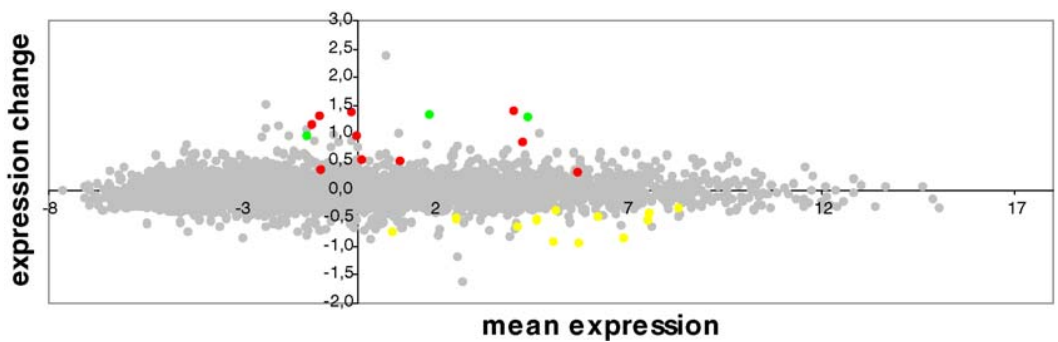

Fig. 4. The expression change in function of the factor sulphur as calculated by a spreadsheet (MS-Excel). The figure shows the genes' expression change in function of the sulphur source against their mean expression. The potentially interesting genes are those away from the main body of the cloud. The highlighted genes are the ones which proved to be of particular interest for the problem investigated by Sekowska et al. (2001); the reader may refer to their work for a detailed discussion. Note that not all of these genes would have been detected using the spreadsheet.

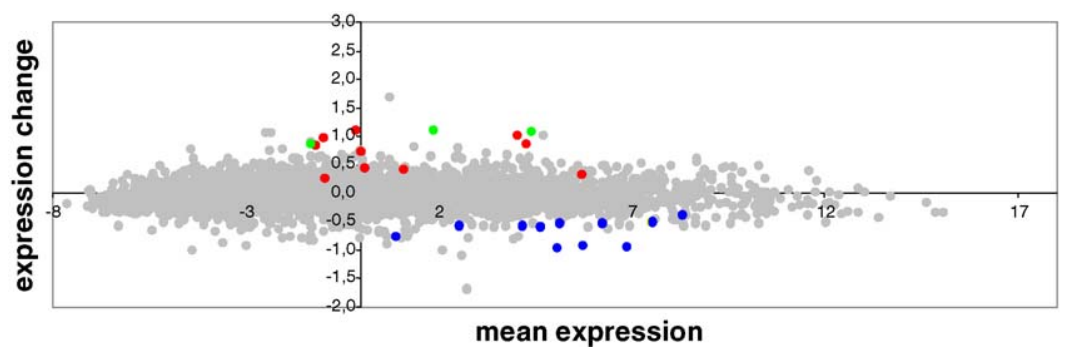

Fig. 5. The data cloud projected on the plane formed by axis 1 against axis 5 (PCA). The figure shows the genes expression change in function of the sulphur source (axis 5) against their mean expression (axis 1), as calculated by PCA. The potentially interesting genes are those away from the main body of the cloud. The highlighted genes are the ones which proved to be of particular interest for the problem investigated by Sekowska et al. (2001); the reader may refer to their work for a detailed discussion. Note that not all of these genes would have been detected using PCA.

The eigenvector matrix gives us also another information: the variance or eigenvalue for each axis, expressed in percentage. This provides an indication of the cloud's dispersion along the axis (the bigger the value, the more the genes are dispersed along this axis). The fundamental idea is that if the dispersion is great, the image is easier to interpret than if all the points were packed together. If an experimental factor influences the expression of some genes, the factor will contribute to the dispersion of the cloud and may coincide with one of the axes determined by PCA.

The eigenvector matrix gives a wealth of information. Looking at our matrix, we see that for the first axis all the 16 coefficients have basically the same value; this means that for the first axis, all experimental conditions have the same weight, in other words, the first axis gives us the total expression of each gene, just like with a spreadsheet. This observation is generally true (see Stoyanova et al., 2004).

In each of the other columns (axes), the experimental conditions can be grouped together according to the sign of their coefficient (positive or negative). For some axes, this coincides with a separation of the two states of a factor. In our case, axis two separates well the two protocols $(1 \mu \mathrm{g}$ RNA: all values are negative and $10 \mu \mathrm{g}$ RNA: all values are positive); axis three separates the day (A and B), axis five the sulphur source (met and $\mathrm{mtr}$ ) and axis seven the two spots (a and b). Other axes, on the other hand correspond to combinations of the experimental conditions, whose interpretation is not evident: axis four is an example. It singles out the ribosomal proteins; a biologically speaking coherent result, which is waiting for an interpretation. This is something frequently found when analyzing microarray data.

The eigenvector matrix deserves a little more attention: the values it contains can be looked at from a different point of view. If we take up our example, each line represents an experimental condition and the values in the 16 columns for a given line give us the position of that particular experimental condition in the 16-dimensional space. (To be precise, each value has to be multiplied with the root of the variance of

Table 1 (Continued)

The mixing matrix at the top was calculated by the spreadsheet, the mixing matrix (or eigenvector matrix) at the bottom by PCA. The arrows indicate the columns which separate well the effects of the same factors. The matrices allow us to change from the old to the new reference frame: they give us for each of the new axes (the columns) the coefiicient with which we have to multiply each gene's value in a given experimental condition (the lines) in order to obtain the new coordinates. The first line in the eigenvector matrix contains the eigenvalue for each axis (in \%), providing an indication of the cloud's dispersion along that axis. Note that for the first axis all the sixteen coefficients have basically the same value; this means that for the first axis, all experimental conditions have the same weight, in other words, the first axis gives us the total expression of each gene, which is generally true (see Stoyanova et al., 2004). An example for the calculation of the new coordinates with the eigenvector matrix: in the original (or "old") reference frame, the gene galK has the coordinates (5.431; $5.432 ; 5.092 ; 5.068 ; 4.893 ; 4.744 ; 3.763 ; 3.661 ; 5.333 ; 5.265 ; 5.329 ; 5.249 ; 4.607 ; 4.444 ; 3.806 ; 3.737)$. To obtain galK's coordinate on the new axis 1 . the calculations are as follows: $(5.431 \times 0.250)+(5.432 \times 0.249)+\cdots+(3.737 \times 0.251)=19.0$. The other coordinates are obtained accordingly. 
that axis, in order to obtain the coordinate.) This means that instead of looking at the eigenvector matrix, we can look at the different projections of the experimental conditions in order to figure out which axes separate well the different states of our factors. Once we have established which planes deserve being examined in details, we come back to the projections of the cloud on these planes and pinpoint those genes, which are far away from the main body of the cloud. Fig. 5 shows the cloud projection on the plane formed by axis one versus axis five.

Note: the normalization of the data is an integral part of PCA.

To resume, with PCA the experimental conditions are not given the same weight (contrary to a spreadsheet) and the criterion chosen to look at the cloud is to maximize the variances along the axes.

\subsubsection{ICA}

"ICA tries to find a linear representation of non-Gaussian data so that the components (or factors, or sources) are statistically independent, or as independent as possible" (Hyvärinen and Oja, 2000).

This search for statistical independence is generally very difficult and therefore an approximation is made: one looks for the directions that maximize the criterion of non-Gaussian distribution. As "non-Gaussian" is a "non-property", numerous possibilities exist for defining such a distribution. One criterion that seems to work quite well is to look for distributions with a positive kurtosis (distributions with "heavy tails"). ICA can be seen as a close relative of PCA. Whilst PCA looks at which directions maximize the variance, ICA approaches the question of finding genes with an "atypical behaviour" more directly, by defining "atypical" as "following a non-Gaussian distribution". The new reference frame will maximize the criterion of "non-Gaussianity". With this criterion, one increases the weight of points that had only small deviations from the main body of the cloud and thus allows them to be detected as potentially interesting.

A latent difficulty with ICA is that there is no analytical solution (contrary to PCA): we look for the numerical solutions. There is the danger that the algorithm finds a direction with a solution, but that this direction is not the best solution

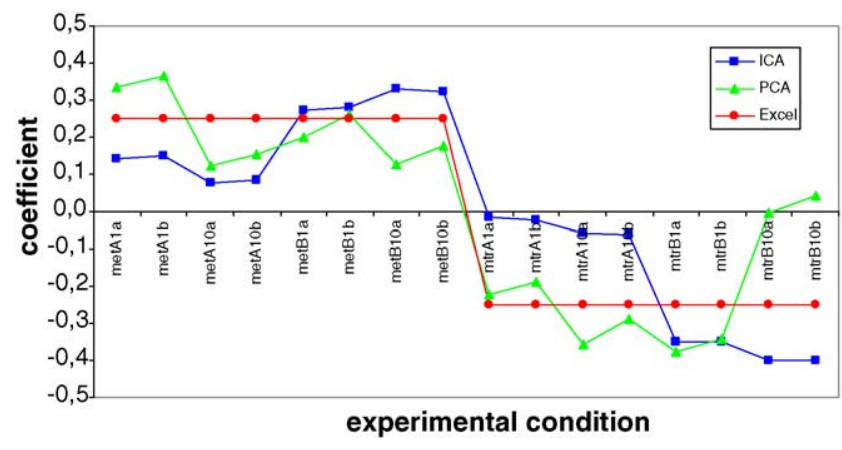

Fig. 7. The "weight" attributed to each experimental condition by a spreadsheet (MS-Excel), PCA and ICA. The figure shows that a spreadsheet (MS-Excel) attributes the same "weight", or importance, to each experimental condition, whilst PCA and ICA do not.

in absolute terms: the algorithm gets stuck with a local maximum (Chiappetta et al., 2004). Launching ICA a large number of times, typically 100, circumvents this problem and only those directions or solutions that have been most frequently found are kept. As with PCA we have a mixing matrix that allows us to change from the old reference frame to the new one. Again, the different experimental conditions do not have the same weight; the weight attributed, though, varies slightly from PCA. Once we have determined the axes, the procedure is the same as with PCA. Fig. 6 shows the cloud projection on the plane that separates well the sulphur sources.

The applications of ICA in microarray analysis include the identification of groups of genes implicated in cancer, the study of the cell cycle (Liebermeister, 2002; Martoglio et al., 2002) and the identification of genes that are potentially coregulated (Chiappetta et al., 2004). Chiappetta et al. (2004) and Carpentier et al. (2004) have applied both PCA and ICA to the sulphur metabolism data and shown that the two methods perform similarly well, with ICA slightly outperforming PCA.

\subsubsection{A brief remark}

We have said that whilst a spreadsheet attributes to each experimental condition the same weight, PCA and ICA do not (Fig. 7 shows a comparison between the three methods from this point of view).

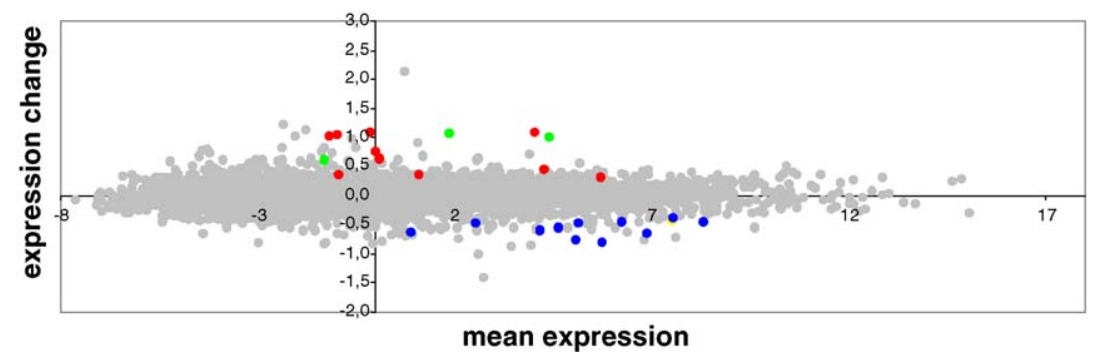

Fig. 6. The data cloud projected on the plane that separates well the sulphur source (ICA). The figure shows the genes' expression change in function of the sulphur source, as determined by ICA. The potentially interesting genes are those away from the main body of the cloud. The highlighted genes are the ones which proved to be of particular interest for the problem investigated by Sekowska et al. (2001); the reader may refer to their work for a detailed discussion. Note that not all of these genes would have been detected using ICA. 
The spreadsheet simply calculates the mean expression. This choice is not optimal when certain experimental conditions contain more information than others. Ideally, the weight attributed should be proportional to the information contained. PCA is a good choice when the signal follows a Gaussian distribution, whilst ICA imposes itself when the distribution is non-Gaussian.

You might wonder what happens if you use, say, PCA on data that follow a non-Gaussian distribution. The answer is that you are likely to miss out on potentially interesting genes; you do not, however, risk finding "wrong" genes. Using more than one tool amounts to examining the cloud from different angles; the results obtained with the different tools are complementary.

\subsection{Statistical tests}

\subsubsection{Preliminary considerations}

Our experience shows that some confusion reigns regarding the statistical tools in general and their application to microarrays in particular. Hence this rather long introductory section.

When approaching microarray data from a statistical point of view, people seem to worry a lot about the fact that the data are "relative" and whether they should or not take ratios.

Microarrays give us "relative data": the interesting information regarding a gene is "relative" as one compares the expression of a gene under condition $\mathrm{A}$ with that of the same gene under condition B. Microarray technology is quite recent; however, dealing with relative data is not and taking the ratio results in a reduction and a falsification of the information offered (Kerr and Churchill, 2001). It is Fisher who first tackled and solved the problem at the very beginning of the 20th century, resulting in ANOVA. For a more detailed discussion of this issue, the reader is referred to the work of Kerr and Churchill (2001). At about the same time, Gosset ("Student") came up with the $t$-test as a solution to the problem.

Statistics help us to answer the question whether the expression differences observed are real. The answer is given indirectly, as the statistical tools give us the probability of having a false positive. A false positive is a gene whose expression difference surpasses by chance a threshold value, which has been fixed in advance. "By chance" means that if the experiment were repeated, you would not find again such a large expression change.

The statistical analysis is used to evaluate the probable percentage of false positives beyond a given threshold value: 40 genes will surpass by chance the threshold value of $1 \%$ if the experiment was carried out on 4000 genes.

The estimation of the number of false positives is only the first step. Beyond the threshold value we not only find false positives, but also genes whose expression change is "real" (we would find it again if the experiment were repeated). The key information is the proportion of false positives on the total, because it measures the risk of being on the wrong track when deciding to work on one of the genes from this group (Benjamini and Hochberg, 1995). One generally chooses the threshold in order to have less than 5\% of false positives in the group. Take for example an experiment carried out on 4000 genes with 80 lying beyond the threshold of $0.1 \%$. As there are on average four false positives beyond the $0.1 \%$ threshold $(4000 \times 0.001)$, the percentage of false positives is $4 / 80$, or $5 \%$ of the selected genes.

The literature sometimes refers to the Bonferroni correction. This correction is not pertinent for the analysis of microarray data, as it is too restrictive.

The numerical criterion used in the statistical tests is always the ratio between the deviations observed for the factor of interest (the signal) and the deviations due to all the causes one chooses to ignore (the noise). The statistical tests differ from each other in the way they define the noise and the probability function they use to estimate the probability of false positives. In the past, the function used was the Gaussian. Nowadays one tends to employ the probability function, estimated on the data using permutations (see Tusher et al., 2001).

\subsubsection{ANOVA}

ANOVA is a tool that allows us to analyze simultaneously the effect of more than one factor on a variable, in our case the genes' expression levels. The method is based on the calculation of the sum of squares, degrees of freedom, mean square (short for mean square deviation from the mean) and $F$-statistics ${ }^{1}$ (see Zar (1998) for details). As we use ANOVA in a somewhat reductive manner, the reader may refer to the work of Zar (1998) for a full appreciation and pedagogic explanation of the possibilities offered.

Various quantities are used simultaneously in order to decide whether the expression of a gene varies significantly for the factor of interest.

1. V1, the variance for the total of the observations made on the gene;

2. V2, the variance for the observations made for the factor of interest;

3. V3, the variance for the observations made for those factors whose influence one wishes to subtract.

The signal is equal to $\mathrm{V} 2$, the noise to $\mathrm{V} 1-(\mathrm{V} 2+\mathrm{V} 3)$. The possibility to calculate the term $\mathrm{V} 3$ is a particularity of ANOVA and it allows a finer control of the noise's composition. In our example, V3 corresponds to the expression change caused by the day, the duplicate and the RNA concentration. The noise encompasses all which causes the difference between the actual expression level and the sum of the expression levels of the four factors.

In the case of the sulphur metabolism data, the equation used for each gene is the following:

$$
Y_{i j k l}=\mu+S_{i}+J_{j}+C_{k}+D_{l}+\varepsilon_{i j k l}
$$

\footnotetext{
${ }^{1}$ Sometimes referred to as $F$-test.
} 


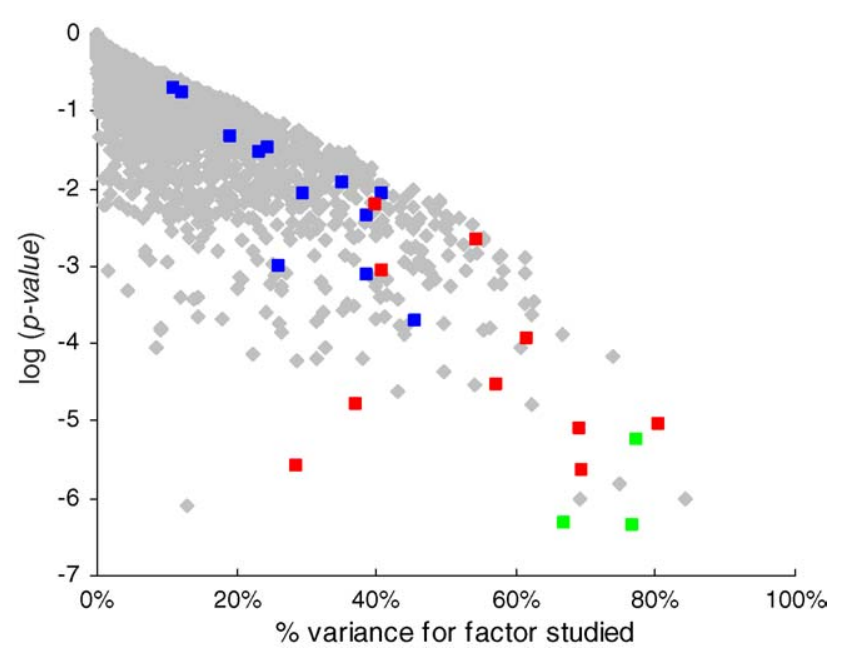

Fig. 8. The graphical representation of the results obtained with ANOVA for the factor sulphur. The potentially interesting genes are those with a small $p$-value and a large variance, genes which are therefore in the bottom right part of the image. They are away from the main body of the cloud. The highlighted genes are the ones which proved to be of particular interest for the problem investigated by Sekowska et al. (2001); the reader may refer to their work for a detailed discussion. As can be seen, not all genes of interest would have been identified by the sole use of ANOVA.

where $Y_{i j k l}$ is the total expression level measured; $\mu$ the mean of the expression levels measured for the gene; $S_{i}, J_{j}, C_{k}$ and $D_{l}$, respectively, the effects of sulphur source $i$, experiment day $j$, RNA concentration $k$ and duplicate $l$ on the expression level; $\varepsilon_{i j k l}$ is the residual error.

Note that the residual error $\varepsilon_{i j k l}$ encompasses all interactions: between two factors (6), between three factors (4) and between four factors (1). The interactions are grouped together under "error" for the following reason: it is information with which we cannot work, unless we have a very precise idea of the nature of the interaction (linear, sinusoidal or other).

The $F$-test is calculated in the following manner: $F=$ "mean square of the sulphur source"/"mean square of the residual error". We are interested in genes that posses a high $F$-value ( $p$-value) for the factor sulphur source. The calculations are done for all genes and the results can be represented in a graphical form. The variance of the factor of interest is given on the $x$-axis, the $p$-value on the $y$-axis. The $p$-value is used to calculate how many false positives will lie below a chosen threshold value (see Fig. 8).

Note that we are not interested, whether the expression levels of the thus identified genes also vary in function of the other factors. One does not preclude the other and has no impact on our analysis.

ANOVA has some advantages when the experimental factors are not binary; in that case, it basically becomes the only tool which is easy to use.

\subsubsection{Paired $t$-test}

We have said that ANOVA quantifies the contribution given by each factor to the total expression of a gene, per- mitting us to isolate the contribution of our factor of interest. The paired $t$-test also allows this, but the approach is different, and we can only use it for binary factors. The paired $t$-test eliminates the influence of all the factors we are not interested in by calculating the difference between pairs of values. The members of each pair differ from each other only with respect to the factor of interest (state 1 versus state 2), all other experimental conditions being equal.

For example, we calculate the difference between the value obtained on met with the value obtained on $\mathrm{mtr}$, both obtained on day A, with $1 \mu \mathrm{g}$ mRNA and spot a. Then we calculate the difference of met versus mtr on day B, with $1 \mu \mathrm{g}$ mRNA and spot a and so forth. This is done for each gene and we thus obtain eight comparisons, or differences per gene. V1 is calculated on these eight comparisons, the term V3 has disappeared.

However, as the paired $t$-test takes pairs of "similar conditions", systemic biases due to, e.g. "day" or "duplicate", are eliminated, therefore still allowing for a reasonable estimation of the error.

\subsection{4. $t$-Test}

The $t$-test corresponds to an ANOVA with one factor and is the least favourable option. The $t$-test only considers the expression difference due to one factor, ignoring that there are pairs of measurements which have more or less in common (like the day, protocol and spot), unlike ANOVA and the paired $t$-test. Thus, we cannot separate the contribution made by our factor of interest from the contribution made by the other factors and the interaction between them; the expression difference due to our factor risks being drowned by the rest.

In terms of V1, V2 and V3: V1 is calculated on the total of the 16 observations made (as with ANOVA), but as the term V3 has disappeared, the noise risks being much larger.

\subsubsection{In conclusion}

The biggest difficulty is to estimate the noise with accuracy. The best solution is to repeat the experiment a large number of times. As this is not always possible, statisticians try to improve the estimation of the noise by working on groups of genes having more or less the same level of noise. A considerable amount of literature is dedicated to this effort. Numerous are the solutions proposed, none is perfect. Generally, the grouping is done a posteriori, after a first estimation of the noise for all the genes separately. One speaks in this case of a Bayesian approach. The reader is referred to the work of Neuhäuser and Senske (2004) for an introduction into the subject and to the work of Kutalik et al. (2004) for the comparison of some methods proposed.

Regarding the three approaches discussed above: Table 2 shows the measurements obtained for $y$ tmJ and the results obtained from ANOVA, the paired $t$-test and the $t$-test. It shows that though ANOVA and the paired $t$-test both identify the gene as interesting, the $t$-test results inconclusive. The observation made on this particular example can be 
Table 2

Comparison between ANOVA, the paired $t$-test and the $t$-test, an example

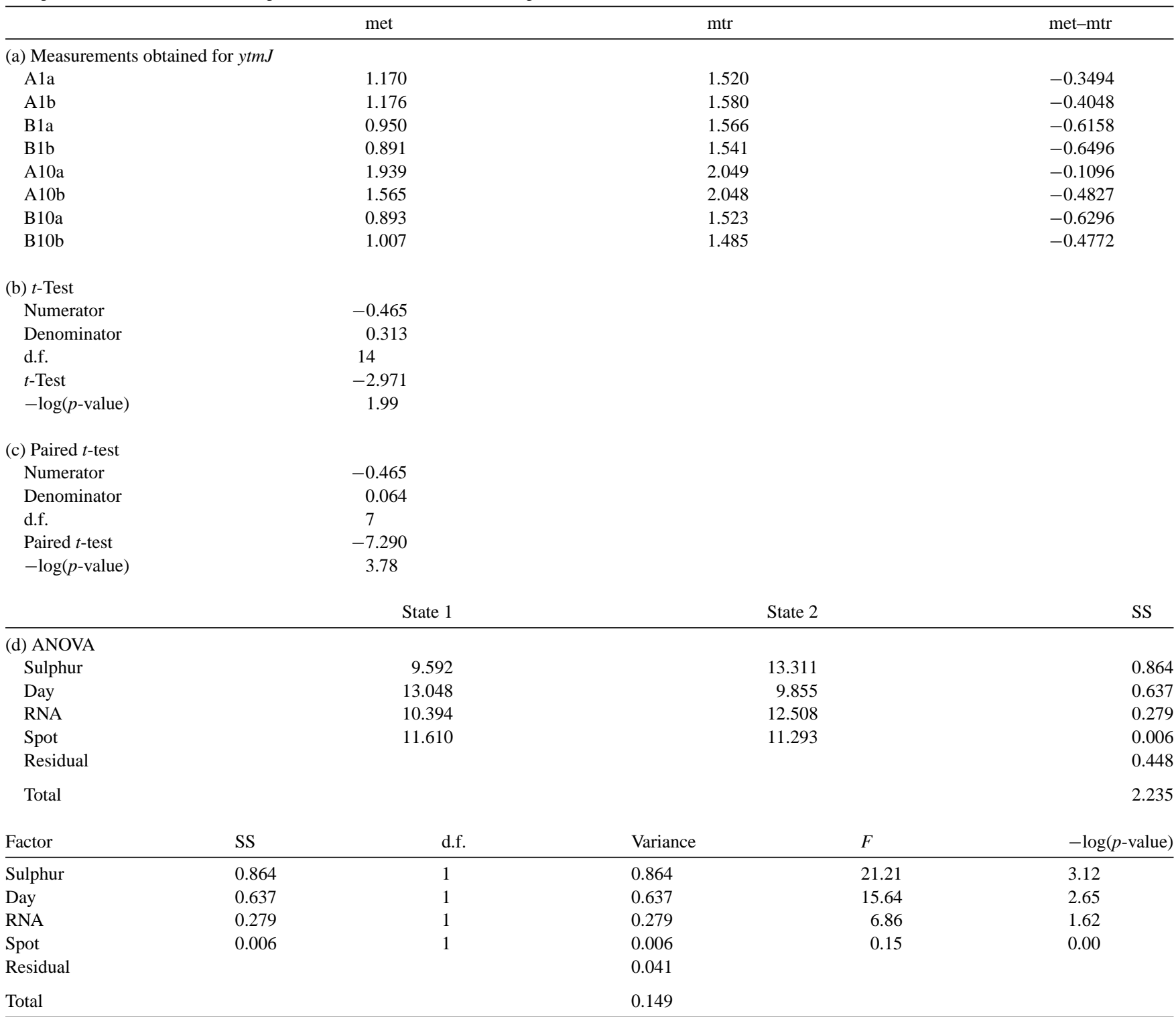

In (a) the measurements obtained for $y$ tmJ are shown. (b-d) The calculations and results obtained with the $t$-test, the paired $t$-test and ANOVA, respectively. ANOVA and the paired $t$-test both identify the gene as potentially interesting, whilst the $t$-test results inconclusive (see the relative $-\log (p$-value)). d.f. $=\operatorname{degrees}$ of freedom; SS = sum of squares. See Section 3.4 for details.

generalized. Table 3 shows a comparison of the number of genes detected by the three methods. Although ANOVA detects the highest number of genes, the paired $t$-test performs comparably well, whilst the $t$-test lags far behind.

\subsection{Graphic exploration and statistical tests in comparison}

We have chosen to talk about the typical representatives of the two approaches. They are not the only ones proposed in the literature: the number of tools is continuously increasing and no one, definitive method has so far emerged, as is exemplified by the web-site maintained by $\mathrm{Li}$, which has a steadily growing collection of articles on microarray data anal- ysis (http://www.nslij-genetics.org/microarray/). Conceptually, all these tools are based on one of the methods described above or they fall into the category "cluster analysis", described below.

Some methods will use the term "distance", whilst others may talk about "correlation". In mathematical terms, it boils down to the same thing: second order statistics, yielding the same type of information. As the methods all differ more or less from each other, it is normal that they do not come up with exactly the same results.

Which method is the best? Carpentier et al. (2004) have examined this issue and developed a protocol that allows the comparison of the different methods, in terms of their reliability. They conclude that each of the methods analyzed gave 
Table 3

Overall comparison between ANOVA, the paired $t$-test and the $t$-test

(a) Number of genes detected with a threshold of $-\log (p$-value $)=3$ ANOVA only 62

$t$-Test only 5

Both 24

ANOVA only $\quad 35$

Paired $t$-test only $\quad 30$

Both 51

$t$-Test only 12

Paired $t$-test only $\quad 64$

Both 17

Total ANOVA $\quad 86$

Total paired $t$-test $\quad 81$

Total $t$-test 29

(b) Number of genes detected with a threshold of $-\log (p$-value $)=4$ ANOVA only 16

$t$-Test only 0

Both 9

ANOVA only 17

Paired $t$-test only 12

Both 8

$t$-Test only 14

Paired $t$-test only 15

Both 5

Total ANOVA 25

Total paired $t$-test $\quad 20$

Total $t$-test 9

The table shows a comparison of the number of genes detected by the three methods. In (a) the threshold for detection was $-\log (p$-value $)=3$, in (b) it was equal to 4 . Although ANOVA detects in both cases the highest number of genes, the paired $t$-test performs comparably well, whilst the $t$-test lags far behind.

some information not provided by the others, suggesting once more the advantage of analyzing one's data with more than one statistical tool.

ANOVA, one of the methods tested, did not excel on the sulphur metabolism data. However, all factors were binary and ANOVA has the great advantage of being easily applicable in cases where the factors are non-binary. It also has another important property: ANOVA is the only method that forces the experimenter from the beginning to give the experimental setup some thought, to plan it carefully. It is therefore a good practice to think of an experimental setup in terms of ANOVA, even if the data are then exploited by another method (see Section 4.2).

\subsection{And the clustering approach?}

The principle is to group and/or to classify the genes in function of the expression profile obtained under the various experimental conditions.

The cloud is thus divided into a number of clusters, the idea being that a cluster corresponds to a functional class. Choosing a gene of unknown function, one can look to which cluster it belongs and thus draw conclusions about its possible role.

This approach poses problems from two points of views: a biological and a technical one.

From a biological point of view: we have to define what a functional class is and how many there are. These are not banal questions, as exemplified by the fact that even for such a well-studied organism like $E$. coli numerous classifications are proposed (for example SwissProt, EcoCyc, Kegg). Secondly, the functional classes found in the literature tend to be rather large, containing dozens or hundreds of genes, making them too large to permit their exploitation in the wet lab. Thirdly, the clustering methods normally do not allow a gene to be part of more than one cluster, which goes against biological intuition and experience.

From a technical point of view: we have to choose amongst a myriad of (family of) clustering techniques. As the biological question is not clearly defined, we do not have a criterion to select the pertinent and coherent method for our needs. ${ }^{2}$ At this point one has to make do with a data-driven attitude. This necessitates a thorough knowledge of the different families of clustering techniques in order to make the best choice in function of the data set to be analyzed (Somorjai et al., 2003), as all the clustering techniques require many prior decisions (Chiappetta et al., 2004). In addition, as Somorjai et al. (2003) point out: "the maxim 'simpler is better' has mostly been ignored".

As clustering methods are well-liked tools (see for example the popular software proposed by Eisen et al. (1998)), various attempts have been made to circumvent the various technical problems. The reader, who would like to have a critical introduction to different families of clustering techniques, may refer to the works of Datta and Datta (2003), De Smet et al. (2002) and Somorjai et al. (2003).

\subsection{And SOM?}

Generally speaking, only the outskirts of the cloud are visually exploitable. The internal organization is hidden by the superposition of thousands of genes on the same image. The analysis would be easier if it were possible to give a faithful representation of the genes' density in each region of the cloud, with only $k$ points. A rather naïve solution consists in choosing these $k$ genes at random. This is unlikely to give satisfactory results, though. Calculating the optimal position of the $k$ points is a difficult problem. A number of programmes exists proposing approximate solutions. An example is self organizing maps (SOM), which chooses the $k$ genes and provides a list of the genes close to the $k$ genes. The interested reader may refer to the work by Kaski et al. (2003) for an

\footnotetext{
2 An example is the definition of the distance between clusters. This is not a banal problem. Take for example the problem of having to define the distance between two countries: do you take the two capitals? The two biggest cities? The shortest distance ( 0 if the countries are adjoining)?
} 
introduction to SOM as well as a comparison of its merits compared to some classic classification methods.

Note that all the programmes proposed necessitate the adjustment of numerous parameters for which you do not necessarily have a rational basis to make your choice. This carries the risk that you only believe those results which tell you something you already know: not the best way to discover new things.

\section{Intricacies of microarray-based methods}

\section{1. $F A Q$}

Over the years of teaching the course on the analysis of microarray data, we have noticed that certain questions, more or less closely related to the subject, turn up on a regular basis. Here are some of them, with the answers.

\subsubsection{Missing values}

They have generally two possible origins: (i) the microarray contains a defect resulting in the impossibility of taking a reading or (ii) the machine eliminates the measurement as the value is very close to the noise level (in this case it would be advisable to change the setup of the machine). This poses a problem, as many data analysis methods require full sets of data. The most radical solution is to eliminate the genes with missing entries, which is obviously far from ideal. A more moderate solution is to fill in the gaps with estimate values. The easiest is to use the row average; the two most common methods, however, are:

(a) looking with whom the "missing gene" associates with in the other experimental conditions, i.e. determining that gene's "neighbours", then presuming that in the missing experiment this gene still associates with them and filling the gap with the median value (a method known under "K-nearest neighbours");

(b) variations around the PCA (examples here are the singular value decomposition and Bayesian principal component analysis (Oba et al., 2003)).

The interested reader is referred to the works of Ouyang et al. (2004), Kim et al. (2004) and Zhou et al. (2003) for the comparison of some currently used estimation methods.

\subsubsection{The correction of the background noise on the membranes, glass plates or silicon chips}

This problem tends to be given too much importance. ANOVA allows us to easily quantify the inter-array variation and the result is that this variation is small compared to other sources (for example "day" in the case of the sulphur metabolism data, see Chen et al. (2004) for a detailed discussion on this subject), strongly suggesting that the effort spent correcting background noise is not justified. There is also a second aspect to be considered, namely that finding a reliable method to correcting background noise is not easy.
As Lawrence et al. (2004) point out, the human component plays an important role. The assumption that the background level is consistent between the DNA spot and the surrounding space, frequently used for background quantification, is not correct (Konishi, 2004). Using "designated" household genes for the background determination is in itself a good idea, but finding out who the household genes are, is posing problems (Stoyanova et al., 2004).

Regarding Affymetrix's GeneChips, the common practice of subtracting the mismatch (MM) probe intensities from the perfect match (PM) ones is "unjustifiable", according to Sasik et al. (2002), as the target sequence hybridizes not only with the PM but also with the MM probe.

We remind the reader at this point that a useful way to assess the utility of an anti-noise measure taken is to check on the change of the eigenvalue of the first axis in a PCA (should increase) or the $F$-value in an ANOVA (should increase).

\subsubsection{Dealing with data containing a large number of very small or zero values}

You may find yourself in the situation of not having access to the "real data": you are given a set of data, where all values below a certain threshold were replaced with one or very few arbitrary values. This means that the distribution is far from being Gaussian (or just unimodal), a fundamental prerequisite for the analysis of the microarray data.

The only solution to this dilemma is to try to "restore" the Gaussian distribution by replacing the smallest values with random values (see Chiappetta et al., 2004).

Having a large number of very small or zero values may simply be the result of a translation, usually the effect of having subtracted the background noise. In this case, it is sufficient to add to all values a constant (for example the weakest signal measured in the experiment) before taking the log of the data.

\subsubsection{Taking the ratio or not?}

Microarrays give us "relative data": the interesting information regarding a gene is "relative" as one compares the expression of a gene under condition A with that of the same gene under condition B. Microarray technology is quite recent; however, dealing with relative data is not. The following text is taken from Kerr and Churchill (2001) who discuss the issue in a very clear and lucid manner: "... relative data is about as old as statistics itself. The "grandfather" of statistics, R.A. Fisher, worked with agricultural field trials. In controlled experiments with clear objectives, scientists sought to determine the productivity of different varieties of a crop, for example different strains. They recognized that there is no such thing in absolute terms as the yield of a variety because productivity depends on soil fertility, sunlight, rainfall, and myriad other factors. They understood that the only meaningful direct comparisons are for strains grown on the same block of land. Consider a hypothetical experiment to study three varieties. Suppose there are three blocks of land available, but each block only has room for two varieties..... 
It is easily accepted that the yield data contain information about the varieties grown in the same block. However, there is a corresponding fact relying on the same logic that can be overlooked. Namely, there is also information about the blocks of land because they have varieties in common. Fisher recognized this duality and realized one could simultaneously estimate the relative yield of varieties and the relative effects of the blocks of land. The quantitative tool for doing this is a simple linear model:

$y_{i j}=\mu+B_{i}+V_{j}+\varepsilon_{j}$

where $y_{i j}$ is the measured yield for variety $j$ grown on block $i$; $\mu$ the overall mean; the block effect $B_{i}$ is the effect of block $i$; and $V_{j}$ is the effect of variety $j$. The term $\varepsilon_{i j}$ represents random error. In a large experiment with many varieties and blocks, unbiased yield comparisons can be made, even for varieties not grown on the same block of land. Returning to microarrays, consider the spots for a particular gene on different arrays (or reproduced within arrays). The spots vary in size, shape, and concentration, analogous to the variation in fertility of blocks of land. Using the same principles as in the agricultural experiment, we can simultaneously measure the relative transcription level of the corresponding gene and the "fertility" of the spots. However, this is only possible if we use all the information in the data and do not reduce to ratios."

This should answer the question adequately.

\subsubsection{The problem posed by the two fluorescent dyes used with glass plates}

When working with glass plates, one is given the choice between two different dyes to be used for the incorporation in order to obtain the hybridization mixture. In other words, one is given the choice between two experimental protocols. In the case of the sulphur metabolism experiment the choice was between using 1 or $10 \mu \mathrm{g}$ mRNA. When ordering the genes as a function of the average intensity of the signal, Sekowska et al. (2001) observed that the order is highly sensitive to the protocol; on the other hand, if the same protocol is used, the results are highly repeatable (see Tables 5 and 6 in Sekowska et al., 2001). This observation can be generalized.

Does this have an impact for the analysis? The factor protocol is an important source of variability (Chen et al., 2004; Sekowska et al., 2001), but as there is no interaction between the other factors, the impact on the analysis is minimal with all the techniques described here (PCA, ICA, ANOVA and paired $t$-test). Having two protocols, doubles the number of measurements without being instructive on the biological problem studied. If one has the means (economic or other) to increase the number of measurements, it is perhaps advisable to introduce a biological repetition or a new biological factor (Chen et al., 2004).

\subsubsection{How many genes should I put on my microarray?}

The answer is simple: as many as possible. The reason is the following: a number of analysis methods normalize the data, which is only justified if three conditions are fulfilled (see "The spread sheet and some preliminary considerations and manipulations" above): firstly, more than $90 \%$ of the genes do not change expression in function of the different experimental conditions, secondly, the number of genes analyzed is large and thirdly the overall intensity change of upand down-regulated genes is similar (see Stoyanova et al., 2004).

\subsubsection{What can I do if my signal is outside the linear range (of my machine)?}

This results in a "cigar" which is twisted and bent. The first and obvious recommendation is to make sure that at the moment of taking the readings, the scanning settings are correct, which they are often not (Stoyanova et al., 2004). The second is to check that one is not just working at one extreme of the linear range; if that is the case, a change of concentration in the hybridization solution is a good option. If the entire linear range is taken up, two solutions can be proposed: using two different voltage settings for the photomultiplier or using different exposure times, when working with radioactively labelled samples. Algorithms for subsequently combining the different readings are readily available (see for example Querec et al., 2004; Lyng et al., 2004). The article by Lyng et al. (2004) shows the relationship between the type of incorrect setting and the resulting cloud shape.

Numerous authors propose "remedies" if the above suggestions prove impossible to follow, but none will give you the "perfect" data back you would have had if the experiments had been executed correctly.

\subsubsection{How does one tackle a temporal series?}

In time series expression experiments a number of samples is taken over a period of time. Biological and computational problems specific to this type of experiment have to be faced from the experimental setup to the data analysis and to the interpretation of the data. The reader is referred to the work by Bar-Joseph (2004) who reviews these problems and the solutions offered.

\subsubsection{How do we find genes for an accurate diagnosis of a disease?}

Typically, the data will come from one hospital and from a relatively small number of patients. These patients represent the learning set and the analysis of the data will always come up with some candidate genes. To validate the results, however, we need a validation set. It is wise to have five to six times more patients in this set than candidate genes. To avoid finding genes that are only specific to a particular sociocultural-genetic background, the patients should be chosen from more than one hospital and more than one country (see also Section 4.2.1).

From a theoretical point of view, the use of microarrays for the diagnosis of a diseases poses two fundamental problems, the first one being Bellman's "curse of dimensionality" (too many features or dimensions, e.g. thousands of genes), the 
second one being the "curse of dataset sparsity" (too few samples) (Somorjai et al., 2003); this means that we end up analyzing a space with a great number of dimensions which is nearly empty: whatever method is applied to the analysis of the data, the result is unlikely to be statistically sound, the biological interpretation risks being inconclusive.

Somorjai et al. (2003) discuss this problem in detail. Hwang et al. (2002) propose a power analysis method in order to determine the minimum sample size for the - statistically reliable - discrimination of distinct disease states.

\subsubsection{How do we determine the relative importance of a factor?}

By using an ANOVA, as it explicitly estimates the magnitude of the sources of variation and therefore gives us the relative importance of each factor (see also Chen et al., 2004).

\subsubsection{What does the p-value tell me? What about false positives?}

The $p$-value gives us the probability of finding by chance a deviation from the mean equal to or larger than the one we observe.

For example, if we decide to work on all those genes with a $p$-value smaller or equal to one per mille $(0.1 \%)$ and we examine 4000 genes, we expect on average 4 genes to fulfil this criterion by chance, without reflecting a biological reality. These four genes fall into the category of false positives. With a $p$-value equal to $5 \%$, we would expect 200 genes to fulfil the criterion by chance.

This means that the $p$-value helps us to judge and quantify the risk of looking at or working with a false positive, nothing more and nothing less.

\subsubsection{Will I not miss out on a few genes?}

Through the years of teaching, we have noticed that this is apparently a worry common to a lot of people working on microarrays.

Even provided that the experiments were planned and executed in a diligent manner, the problem will generally not be that of having too little genes changing expression, but too many. An example is given again by the sulphur metabolism experiment where less than a dozen genes were of actual interest, truly involved in the phenomenon studied, but a lot more changed expression. The reason for this "surplus" of genes is that there will always be secondary effects. Biological processes seldom come in a straight line; more often they resemble an intricate net (think of the cell's metabolism), which means it is near impossible to isolate a phenomenon completely (see Sontag et al., 2004). To further narrow down the list of candidate genes, one will have to use any available (biological) knowledge from other sources.

\subsection{How to plan one's experiment?}

It is quite usual to find that a rather large number of genes, typically around $10 \%$, change expression consider- ably between two experimental conditions. This number is too large to be directly exploitable and we will have to extract a short and pertinent list of genes to work with. This task is greatly facilitated by an adequate and well thought-through experimental setup.

\subsubsection{The type of factors}

An experiment is made up of three types of factors, each providing specific information.

The first factor corresponds to the phenomenon studied. The study concern two or more states (two culture conditions, for example, or a certain number of samples taken during a time course experiment). The aim is to narrow down to a maximum the target genes, in other words to have only few genes who change expression considerably between the different experimental states. For this, the experimental states should be as close as possible, for example:

(a) In the case of the sulphur metabolism experiments, the two sulphur sources were metabolically speaking closely related.

(b) When trying to isolate genes typical of a certain cancer, one should study different subtypes, all closely related to the one of interest.

If this maxim is not observed, too many genes will change expression considerably and the identification of target genes will become near impossible.

The second type of factor serves to verify whether the observations made hold true if the biological parameters are changed. Do we find the same candidate genes if we work with a different bacterial strain? Or patients from a different hospital? Or if the experiment is carried out on a different day? Note that even repeating the experiment on a different date introduces a biological variability, as the experimental conditions will never be exactly the same (see Sekowska et al., 2001). This verification is extremely important as the most interesting genes are those which come up whatever the biological parameters. They are most likely the genes at the heart of the phenomenon studied, as their behaviour is not bound to a particular context (genetic, socio-cultural or physiological). The reader is referred to the works of Turk et al. (2004) and Whitehead and Crawford (2005) who discuss this issue.

The third type of factor is a technical one: the type of protocol used to label the cDNA, having two spots for each gene on the array or using the dye swap. This type of factor increases the workload without adding any biologically pertinent information. The experimental protocols have become highly reproducible and it is advisable to stick to just one protocol (with its systemic biases) and increase the number of states of the two other factors.

\subsubsection{The ideal situation: a fully crossed factorial design}

The best experimental setup is to follow a fully crossed experimental design (exemplified by ANOVA) as it 
(a) allows a good exploitation of the information given;

(b) allows a precise estimation of the error variance (see Fisher (1951) for the original discussion or Mather (1943), Zar (1998) and Kerr et al. (2000) for a more user-friendly approach).

Setting up a fully crossed factorial design means that each level (state) of one factor is found in combination with each level of the other factors, as shown in Fig. 1. Note that carrying out twice the experiment on strain 1 on day A and twice the experiment on strain 2 on day B would not be adequate as it would be impossible to separate the effect of the day from the effect of the strain.

\subsubsection{The reality}

A fully crossed factorial design may not be possible. This is typically the case in clinical studies, as they strongly depend on the hospitals' random recruitment of patients. These represent a learning set. To confirm the results a validation set would be needed, and to avoid finding genes specific to a particular socio-cultural-genetic background only, the recruitment should be made at more than one hospital and more than one country.

This may prove to be unfeasible if not impossible. A different option is to give up on the fully crossed factorial design altogether and take a completely different approach: one can exploit all the experimental data available in the literature (freely available on the web) by pooling them together. This is not as bizarre an idea as it may seem; the aim can be to increase the number of patients (Jiang et al., 2004) or to get information about the co-expression and co-regulation of genes (Lee et al., 2004; Yeung et al., 2004). Especially for the two latter issues, this is the only approach: as a very large amount of data is needed, which a single lab could not possibly come up with.

Various authors propose statistical models to help extracting the maximum information from these pooled data (see for example Shen et al. (2004) and Statnikov et al. (2005)). Note that when working with pooled date, their analysis will have to be carried out with methods which do not need the definition of the factors a priori, like PCA or ICA.

\subsubsection{The combination of factors}

If we want to obtain useful information from our microarray experiment, we are forced to formulate precise questions. This means that we cannot combine two factors in one question, as this is equivalent to measuring the interaction between the factors, which is not separable from the error (unless we know in detail the relationship between the two factors, for example linear or sinusoidal).

This is one more reason to follow a fully crossed factorial experimental setup, as exemplified by ANOVA. It forces us to spell out in detail what we want to measure and what will be part of the error or interaction component. It has the great advantage of permitting the identification of the sources of variability and their magnitude; this allows making the improvements to the setup at the right sources, which will generally be a modification of the experimental protocol and an increase in the number of biological replications (Chen et al., 2004).

\section{The answer to all our questions?}

Microarrays are sometimes seen as the miracle tool, which will give all the answers to all the questions. Paying considerable attention to the experimental setup is a necessary condition, but not a sufficient one.

The preliminary phase should already take into consideration the different analysis options available to the experimenter by pulling in statisticians. This should be an exchange, not a handing over the job to a statistician, as the biological question has to stay at the front. As Vingron (2001) points out in his editorial, bioinformaticians should "go back to school and learn more statistics. Not so much with the goal of mastering all of statistics but with the goal of sufficiently educating ourselves on order to pull in statisticians."

A careful analysis of the data should follow (we suggest using more than one method, as they tend to give complementary information).

The complex nature of biological phenomena means that it is near impossible to isolate the candidate genes only through a microarray experiment (Curtis and Brand, 2004; Somorjai et al., 2003; Sontag et al., 2004), meaning that the list of candidate genes obtained will have to be further worked on. This may be done by further theoretical work (integration of all available biological knowledge) or additional experiments in the wet-lab.

Sometimes the a priori biological knowledge about the phenomenon of interest may be very limited. In this case, it can happen that despite careful planning and execution, the genes identified as interesting are not actually the cause of the phenomenon. This was for example the case in the genomewide analysis undertaken by Oshima et al. (2002). In these cases a new experimental setup may be solution. However, the conclusion may also be that a transcriptome analysis is not the adequate tool for the study of the phenomenon (Riva et al., 2004).

It is therefore important to realize that a microarray analysis will generally not be THE answer to all your questions. It is a complement to other approaches.

\section{Software and data used}

The sulphur metabolism data from Sekowska et al. (2001) are freely accessible at http://195.221.65.10:1234/ $\sim$ carpenti/.

PCA and ANOVA were performed using GeneANOVA, freely available on request for non-commercial use. Please contact Gilles Didier at didier@iml.univ-mrs.fr. 
ICA was adapted to gene expression analysis by Bruno Torrésani, Pierre Chiappetta and Marie-Christine Roubaud (see http://www.cmi.univ-mrs.fr/ torresan/publi.html).

\section{Acknowledgments}

The authors wish to thank J.-L. Risler for critical reading of the manuscript. This work was supported in part by the French Ministry of the Economy, Finance and Industry (contract ASG no. 01490 6093).

\section{References}

Bar-Joseph, Z., 2004. Analyzing time series gene expression data. Bioinformatics 20, 2493-2503.

Barrett, J.C., Kawasaki, E.S., 2003. Microarrays: the use of oligonucleotides and cDNA for the analysis of gene expression. Drug Discov. Today 8, 134-141.

Benjamini, Y., Hochberg, Y., 1995. Controlling the false discovery rate: a practical and powerful approach to multiple testing. J. Roy. Stat. Soc., Ser. B 57, 289-300.

Bussemaker, H.J., Li, H., Siggia, E.D., 2000. Building a dictionary for genomes: identification of presumptive regulatory sites by statistical analysis. Proc. Natl. Acad. Sci. U.S.A. 97, 10096-10100.

Callow, M.J., Dudoit, S., Gong, E.L., Speed, T.P., Rubin, E.M., 2000. Microarray expression profiling identifies genes with altered expression in HDL-deficient mice. Genome Res. 10, 2022-2029.

Carpentier, A.-S., Riva, A., Tisseur, P., Didier, G., Hénaut, A., 2004. The operons, a criterion to compare the reliability of transcriptome analysis tools: ICA is more reliable than ANOVA, PLS and PCA Comput. Biol. Chem. 28, 3-10.

Chen, J., Delongchamp, R., Tsai, C.-A., Hsueh, H.-M., Sistare, F., Thompson, K.L., Desai, V.G., Fuscoe, J.C., 2004. Analysis of variance components in gene expression data. Bioinformatics 20, 1436-1446.

Chiappetta, P., Roubaud, M.C., Torrésani, B., 2004. Blind source separation and the analysis of microarray data. J. Comput. Biol. 11, 1090-1109.

Curtis, R.K., Brand, M.D., 2004. Analysing microarray data using modular regulation analysis. Bioinformatics 20, 1272-1284.

Datta, S., Datta, S., 2003. Comparisons and validation of statistical clustering techniques for microarray gene expression data. Bioinformatics 19, 459-466.

Davis, S.J., Millar, A.J., 2001. Watching the hands of the Arabidopsis biological clock. Genome Biol. 2, e-pub.

De Smet, F., Mathys, J., Marchal, K., Thijs, G., De Moor, B., Moreau, Y., 2002. Adaptive quality-based clustering of gene expression profiles. Bioinformatics 18, 735-746.

Deutsch, J.M., 2003. Evolutionary algorithms for finding optimal gene sets in microarray prediction. Bioinformatics 19, 45-52.

Eisen, M.B., Spellman, P.T., Brown, P.O., Botstein, D., 1998. Cluster analysis and display of genome-wide expression patterns. PNAS 95, 14863-14868.

Ekins, R.P., 1989. Multi-analyse immunoassay. J. Pharm. Biomed. Anal. 7, 155-168.

Ekins, R.P., Chu, F., Biggart, E., 1990. Multispot, multianalyte, immunoassay. Ann. Biol. Clin. (Paris) 48, 655-666.

Ekins, R.P., Chu, F.W., 1991. Multianalyse microspot immunoassaymicroanalytical "compact disk" of the future. Clin. Chem. 37, 1955-1967.

Fisher, R.A., 1951. The Design of Experiments, 6th ed. Oliver and Boyd, London.
Fodor, S.P., Read, J.L., Pirrung, M.C., Stryer, L., Lu, A.T., Solas, D., 1991. Light-directed, spatially addressable parallel chemical synthesis. Science 251, 767-773.

Heyer, L.J., Kruglyak, S., Yooseph, S., 1999. Exploring expression data: identification and analysis of coexpressed genes. Genome Res. 9, 1106-1115.

Hirai, M.Y., Yano, M., Goodenowe, D., Kanaya, S., Kimura, T., Awazuhara, M., Arita, M., Fujiwara, T., Saito, K., 2004. Integration of transcriptomics and metabolomics for understanding of global responses to nutritional stresses in Arabidopsis thaliana. PNAS 101, $10205-10210$.

Hoyle, D., Rattray, M., Jupp, R., Brass, A., 2002. Making sense of microarray data distributions. Bioinformatics 18, 576-584.

Hwang, D., Schmitt, W., Stephanopoulos, G., Stephanopoulos, G., 2002. Determination of minimum sample size and discriminatory expression patterns in microarray data. Bioinformatics 18, 1184-1193.

Hyvärinen, A., Oja, E., 2000. Independent component analysis: algorithms and applications. Neural Networks 13, 411-430.

Jarvis, J., Dozmorov, I., Jiang, K., Frank, M.B., Szodoray, P., Alex, P., Centola, M., 2004. Novel approaches to gene expression analysis of active polyarticular juvenile rheumatoid arthritis. Arthritis Res. Ther. 6, R15-R32.

Jiang, H., Deng, Y., Chen, H.-S., Tao, L., Sha, Q., Chen, J., Tsai, C.-J., Zhang, S., 2004. Joint analysis of two microarray gene-expression data sets to select lung adenocarcinoma marker genes. BMC Bioinformatics 5,81 , e-pub.

Joos, L., Eryuksel, E., Brutsche, M.H., 2003. Functional genomics and gene microarrays-the use in research and clinical medicine. Swiss Med. Wkly. 133, 31-38.

Jordan, B., 2002. Historical background and anticipated developments. Ann. NY Acad. Sci. 975, 24-32.

Kaski, S., Nikkilä, J., Oja, M., Venna, J., Törönen, P., Castrén, E., 2003. Trustworthiness and metrics in visualizing similarity of gene expression. BMC Bioinformatics 4, 48, e-pub.

Kendall, M., Stuart, A., Ord, J.K., 1983. The Advanced Theory of Statistics, vol. 3: Design and Analysis, and Time-series. Charles Griffin \& Co.

Kerr, K., Churchill, G., 2001. Statistical design and the analysis of gene expression microarray data. Genet. Res. 77, 123-128.

Kerr, K., Martin, M., Churchill, G., 2000. Analysis of variance for gene expression microarray data. J. Comput. Biol. 7, 819-837.

Kim, K.-Y., Kim, B.-J., Yi, G.-S., 2004. Reuse of imputed data in microarray analysis increases imputation efficiency. BMC Bioinformatics 5, 160 , e-pub.

Konishi, T., 2004. Three-parameter lognormal distribution ubiquitously found in cDNA microarray data and its application to parametric data treatment. BMC Bioinformatics 5, 5, e-pub.

Kutalik, Z., Inwald, J., Gordon, S.V., Hewinson, R.G., Bucher, P., Hinds, J., Cho, K.-H., Wokenhauer, O., 2004. Advanced significance analysis of microarray data based on weighted resampling: a comparative study and application to gene deletions in Mycobacterium bovis. Bioinformatics 20, 357-363.

Lawrence, N., Milo, M., Niranjan, M., Rashbass, P., Soullier, S., 2004. Reducing the variability in cDNA microarray image processing by Bayesian inference. Bioinformatics 20, 518-526.

Lee, H., Hsu, A., Sajdak, J., Qin, J., Pavlidis, P., 2004. Coexpression analysis of human genes across many microarray data sets. Genome Res. 14, 1085-1094.

Liebermeister, W., 2002. Linear modes of gene expression determined by independent component analysis. Bioinformatics 18, 51-60.

Lipshutz, R.J., Fodor, S.P., Gingeras, T.R., Lockhart, D.J., 1999. High density synthetic oligonucleotide arrays. Nat. Genet. 21, 2024.

Lyng, J., Badiee, A., Svendsrud, D., Hovig, E., Myklebost, O., Stokke, T., 2004. Profound influence of microarray scanner characteristics on gene expression ratios: analysis and procedure for correction. BMC Genomics 5, 10, e-pub. 
Martoglio, A.-M., Miskin, J., Smith, S., MacKay, D., 2002. A decomposition model to track gene expression signatures: preview on observer-independent classification of ovarian cancer. Bioinformatics 18, 1617-1624.

Mather, K., 1943. Statistical Analysis in Biology, 1st ed. Methuen.

McCune, H.J., Donaldson, A.D., 2003. DNA replication: telling time with microarrays. Genome Biol. 4, 204, e-pub.

Neuhäuser, M., Senske, R., 2004. The Baumgartner-Weiß-Schindler test for the detection of differentially expressed genes in replicated microarray experiments. Bioinformatics 20, 3553-3564.

Oba, S., Sato, M.-A., Takemasa, I., Monden, M., Matsubara, K.-I., Ishii, S., 2003. A Bayesian missing value estimation method for gene expression profile data. Bioinformatics 19, 2088-2096.

Oshima, T., Wade, C., Kawagoe, Y., Ara, T., Maeda, M., Masuda, Y., Hiraga, S., Mori, H., 2002. Genome-wide analysis of deoxyadenosine methyltransferase-mediated control of gene expression in Escherichia coli. Mol. Microbiol. 45, 673-695.

Ouyang, M., Welsh, W.J., Georgopoulos, P., 2004. Gaussian mixture clustering and imputation of microarray data. Bioinformatics 20, 917-923.

Park, P.J., Butt, A.J., Kohane, I.S., 2002. Comparing expression profiles of genes with similar promoter regions. Bioinformatics 18, 1576-1584.

Querec, T., Stoyanova, R., Ross, E., Patriotis, C., 2004. A novel approach for increasing sensitivity and correcting saturation artifacts of radioactively labeled cDNA arrays. Bioinformatics 20, 1955-1961.

Renn, S., Aubin-Horth, N., Hofmann, H., 2004. Biologically meaningful expression profiling across species using heterologous hybridization to a cDNA microarray. BMC Genomics 5, 42, e-pub.

Riva, A., Delorme, M.-O., Chevalier, T., Guilhot, N., Henaut, C., Henaut, A., 2004. The difficult interpretation of transcriptome data: the case of the GATC regulatory network. Comput. Biol. Chem. 28, 109118.

Sasik, R., Calvo, E., Corbeil, J., 2002. Statistical analysis of high-density oligonucleotide arrays: a multiplicative noise model. Bioinformatics $18,1633-1640$

Schena, M., Shalon, D., Davis, R.W., Brown, P.O., 1995. Quantitative monitoring of gene expression patterns with a complementary DNA microarray. Science 270, 467-470.

Sekowska, A., Robin, S., Daudin, J.J., Henaut, A., Danchin, A., 2001. Extracting biological information from DNA arrays: an unexpected link between arginine and methionine metabolism in Bacillus subtilis. Genome Biol. 2, Research 0019, e-pub.

Shen, R., Ghosh, D., Chinnaiyan, A.M., 2004. Prognostic meta-signature of breast cancer developed by two-stage mixture modeling of microarray data. BMC Genomics 5, 94 .
Somorjai, R.L., Dolenko, B., Baumgartner, R., 2003. Class prediction and discovery using gene microarray and proteomics mass spectroscopy data: curses, caveats, cautions. Bioinformatics 19, 1484-1491.

Sontag, E., Kiyatkin, A., Kholodenko, B., 2004. Inferring dynamic architecture of cellular networks using time series of gene expression, protein and metabolite data. Bioinformatics 20, 1877-1886.

Southern, E.M., Maskos, U., Elder, J.K., 1992. Analyzing and comparing nucleic acid sequences by hybridization to arrays of oligonucleotides: evaluation using experimental models. Genomics 13, 1008-1017.

Stanewsky, R., 2003. Genetic analysis of the circadian system in Drosophila melanogaster and mammals. J. Neurobiol. 54, 111-147.

Statnikov, A., Aliferis, C.F., Tsamardinos, I., Hardin, D., Levy, S., 2005. A comprehensive evaluation of multicategory classification methods for microarray gene expression cancer diagnosis. Bioinformatics 21 , 631-643.

Stoyanova, R., Querec, T., Brown, T., Patriotis, C., 2004. Normalization of single-channel DNA array data by principal component analysis. Bioinformatics 20, 1772-1784.

Templin, M.F., Stoll, D., Schrenk, M., Traub, P.C., Vohringer, C.F., Joos, T.O., 2002. Protein microarray technology. Trends Biotechnol. 20, $160-166$.

Thygesen, H., Zwinderman, A., 2004. Comparing transformation methods for DNA microarray data. BMC Bioinformatics 5, 77, e-pub.

Turk, R., t'Hoen, P.A., Sterrenburg, E., de Menezes, R.X., de Meijer, E.J., Boer, J.M., van Ommen, G.-J.B., den Dunnen, J.T., 2004. Gene expression variation between mouse inbred strains. BMC Genomics 5, 57, e-pub.

Tusher, V., Tibshirani, R., Chu, G., 2001. Significance analysis of microarrays applied to the ionizing radiation response. PNAS 98, 5116-5121.

Vingron, M., 2001. Bioinformatics needs to adopt statistical thinking. Bioinformatics 17, 389-390.

Vrana, K.E., Freeman, W.M., Aschner, M., 2003. Use of microarray technologies in toxicology research. Neurotoxicology 24, 321-332.

Whitehead, A., Crawford, D.L., 2005. Variation in tissue-specific gene expression among natural populations. Genome Biol. 6, R13, e-pub.

Yeung, K.Y., Medvedovic, M., Bumgarner, R., 2004. From co-expression to co-regulation: how many microarray experiments do we need? Genome Biol. 5, R48, e-pub.

Zar, J.H., 1998. Biostatistical Analysis, 4th ed. Pearson Education.

Zhao, Y., Li, M.-C., Simon, R., 2005. An adaptive method for cDNA microarray normalization. BMC Bioinformatics 6, 28, e-pub.

Zhou, X., Wang, X., Dougherty, E.R., 2003. Missing-value estimation using linear and non-linear regression with Bayesian gene selection. Bioinformatics 19, 2302-2307. 